\title{
Facilitation of classical conditioning in rabbits by reinforcing brain stimulation'
}

ALFRED BRUNER

NATIONAL PHYSICAL LABORATORY, ENGLAND

Ss receiving positively or negatively reinforcing brain stimulation coupled to an eye-shock UCS achieved classically conditioned nictitating membrane responses to a buzzer CS faster than Ss receiving neutral or no brain stimulation.

Two previous classical conditioning (CC) studies have indicated that in order to elaborate reliable conditioned responses (CRs), the UCS must possess motivational properties (Gerall, Sampson, \& Boslov, 1957; Bruner, 1965). The present report further demonstrates the importance of the reinforcement mechanism in $\mathrm{CC}$ by showing that coupling reinforcing brain stimulation (BS) to a conventional UCS significantly hastens $\mathrm{CR}$ acquisition, irrespective of the motivational polarity of the BS.

\section{Method}

The Ss were 33 adult, male, New Zealand White rabbits, 20 of which were stereotaxically implanted with two or more chronic electrodes. At least one week after surgery each implanted $S$ was tested for stimulation effects in a box approximately $2 \times 3 \mathrm{ft}$., having a lever 7 in. wide protruding from one wall, 4-1/2 in. above floor level. Stimulus effects and optimum intensities (which ranged from 40-200 $\mu$ a) were determined for each electrode using a .5-sec.train of $125 \mathrm{cps}$, biphasic, rectangular, constant current pulses, of 1-msec. duration each. One to three $1-\mathrm{hr}$. sessions were required to test the stimulation effects for each $\mathrm{S}$. When a positively reinforcing placement was stimulated approach responses and lever pressing (using chin or mouth) were readily forthcoming, with minimal shaping (response rates were from 20 to over $80 / \mathrm{min}$.; operant rate virtually zero), while startle and escape responses following stimulation constituted the criteria for negatively reinforcing placements. Further details of the BS effects and anatomical localization are the subject of a separate paper in preparation. In brief, self-stimulation was most readily elicited from the postero-lateral hypothalamus and fornix (e.g. P1, L2.5, H-5, Sawyer et al, 1954), while negative effects were most consistently observed in the midbrain reticular formation, medial hypothalamus, and portions of the anterior hypothalamus. Neutral effects were seen in the caudate nucleus, hippocampus, cortex, and elsewhere.

Several days following the BS tests each $S$ was run in the nictitating membrane $\mathrm{CC}$ procedure (Gormezano, 1966) as follows: Day 1-nylon loop sutured in nictitating membrane and wound clips attached $1 / 2$ in. above and below eye; Day 2-1-hr. adaptation to restrainer and membrane connection in running chamber; Days $3,4,5$, and 6-conditioning sessions, 40 trials each; Day 7-80 trials of extinction. The CS was a 1-sec., $70 \mathrm{~dB}$ buzzer. The UCS was the shock discharge of a .1-mfd capacitor charged to $26 \mathrm{~V}$ (approximately 4-10 ma peak, 1-msec. decay time) delivered via the wound clips .5 sec. after CS onset. Experimental Ss received a .5-sec. train of BS simultaneously with eye shock, the BS and CS thus terminating together, at an intensity determined during the pretest. 2 The intertrial interval was $90 \mathrm{sec}$. and constant. Membrane extenstion occurring between 25 and 500 msec. after CS onset were scored as CRs. Results

The number of acquisition trials required to start a criterion run of $8 \mathrm{CRs}$ out of 10 successive trials were: for individual Ss in the positively stimulated group, 23, $37,30,44,29,57,40,38$; for the negatively stimulated Ss, 29, 43, 53, 57; for the unimplanted controls, 84,85 , $104,101,41,88,46,56,84,48,215,140,110$; for three Ss receiving emotionally neutral stimulation, 85, 82, 82; and for five Ss implanted with positive hypothalamic electrodes, but not stimulated during $\mathrm{CC}, 82,103,90,88$, and 93. This criterion method of data expression was chosen over the more conventional learning curves because, when using an eye shock UCS, all rabbits so far observed by this investigator have tended to show stepwise "learning functions," i.e., once the CR appeared it occurred on virtually every trial thereafter, with the first CR appearing very near the start of a session, suggesting an overnight gain's effect (Bruner, 1965).

The effect of coupling positively reinforcing BS to the UCS was to significantly hasten acquisition relative to the unimplanted controls $(p<.01$, Mann-Whitney $U$ test). The negatively stimulated animals also tended to acquire the $\mathrm{CR}$ more rapidly than controls, while the Ss receiving neutral $\mathrm{BS}$ and the non-stimulated implants did not. Although the positively stimulated animals also tended to show greater resistance to extinction, those data were highly variable and therefore not presented here.

To see if positive BS alone would maintain CR strength previously established, four Ss previously conditioned with the eye shock were fully reconditioned the day following extinction. After CR strength had been fully reinstated the eye shock was omitted, leaving only the CS and $B S$. In all Ss the CR weakened and vanished rather quick$1 y$, at the same rateas for controls, failing to support the possibility that the BS could serve as a direct reinforcer for the membrane CR. 


\section{Discussion}

The tendency for the negative BS to facilitate acquisition when coupled with the eye shock could have been predicted on the basis of an increased UCS intensity effect, since both stimulation and eye shock were aversive. But the observation that acquisition was similarly facilitated by positively reinforcing BS is rather surprising in view of the opposite reinforcing polarities of the BS and eye shock. Furthermore, others have found (e.g. Cox \& Valenstein, 1965) that positively reinforcing BS attenuates the aversiveness of peripheral (foot) shock in rats, insofar as discrimination-preference tests reveal. If the only effect of reinforcing $B S$ was to attenuate the aversiveness of the eye shock in the present study, we would expect acquisition to have been retarded, as that is the usual result of reduced UCS intensity. But the present results are clearly at odds with the simplification that emotional stimuli of opposite polarity merely interact so as to neutralize one another. On the other hand, if the rapid acquisition presently observed may be taken to indicate that the UCS event was enhanced by the addition of positive BS, the implication is that the total non-algebraic amount of "emotional activation" triggered by the UCS complex is an important variable regulating $\mathrm{CR}$ acquisition.

\section{References}

Bruner, A. UCS properties in classical conditioning of the albino rabbit's nictitating membrane response. J. exp. Psychol., 1965. 69, 186-192.

Cox, V. C., \& Valenstein, E. S. Attenuation of aversive properties of peripheral shock by hypothalamic stimulation. Science, 1965 , $149,323-325$.

Gerall, A. A., Sampson, P. B.. \& Boslov, Gertrude L. Classical conditioning of human pupillary dilation. J. exp. Psychol., 1957, $54,467-474$.

Gormezano, I. Classical conditioning. In J. B. Sidowski (Ed.), Experimental methods and instrumentation in psychology. New York: McGraw-Hill, 1966.

Sawyer, C. H., Everett, J. W., \& Green, J. D. The rabbit diencephalon in stereotaxic coordinates. J. comp.. Neurol., 1954, 101, 801-824.

\section{Notes}

1. Supported in part by NSF Grants G18891 and GB4363 to E. J. Wyers, and performed at the University of Southern California. 2. Brain stimulation alone never elicited membrane movements. Four additional rabbits receiving four sessnons of CS-positive BS pairings, without eye shocks, manifested no CRs. 